\title{
Is There a "Trial Effect" on Outcome of Patients with Metastatic Renal Cell Carcinoma Treated with Sunitinib?
}

\section{Daniel Keizman, MD \\ Keren Rouvinov, MD \\ Avishay Sella, MD \\ Maya Gottfried, MD \\ Natalie Maimon, MD \\ Jenny J. Kim, MD \\ Mario A. Eisenberger, MD \\ Victoria Sinibaldi, MD \\ Avivit Peer, MD \\ Michael A. Carducci, MD \\ Wilmosh Mermershtain, MD \\ Raya Leibowitz-amit, MD \\ Rony Weitzen, MD \\ Raanan Berger, MD}

Genitourinary Oncology Service, Institute of Oncology, Meir Medical Center, Sackler School of Medicine,

Tel Aviv University, Kfar Saba, Israel

Correspondence: Daniel Keizman, MD Genitourinary Oncology Service,

Institute of Oncology, Meir Medical Center, Sackler School of Medicine, Tel Aviv University, Tshernichovsky 59, Kfar Saba, 44281, Israel

Tel: $972-9-7472714$

Fax: 972-9-7472979

E-mail: danielkeizman@gmail.com

Received October 15, 2014

Accepted December 21, 2014

Published Online March 5, 2015

*Daniel Keizman and Keren Rouvinov contributed equally to this work.

${ }^{+}$Part of the data was presented (poster) at the Genitourinary Cancers Symposium:

The American Society of Clinical Oncology (ASCO), Orlando, February 2013,

and published in abstract form

(J Clin Oncol 2013;31 Suppl 6:abstr 453),

and at the European Multidisciplinary Meeting on Urological Cancers (EMUC), Marseille,

November 2013, and published in abstract form (Eur Urol 2013;12 Suppl:abstr 045).

\section{Purpose}

Studies suggested the existence of a 'trial effect,' in which for a given treatment, participation in a clinical trial is associated with a better outcome. Sunitinib is a standard treatment for metastatic renal cell carcinoma (mRCC). We aimed to study the effect of clinical trial participation on the outcome of $\mathrm{mRCC}$ patients treated with sunitinib, which at present, is poorly defined.

\section{Materials and Methods}

The records of mRCC patients treated with sunitinib between 2004-2013 in 7 centers across 2 countries were reviewed. We compared the response rate (RR), progression free survival (PFS), and overall survival (OS), between clinical trial participants $(n=49)$ and a matched cohort of non-participants $(n=49)$ who received standard therapy. Each clinical trial participant was individually matched with a non-participant by clinicopathologic factors. PFS and OS were determined by Cox regression.

\section{Results}

The groups were matched by age (median, 64), sex (male, 67\%), Heng risk (favorable, 25\%; intermediate, 59\%; poor, 16\%), prior nephrectomy (92\%), RCC histology (clear cell 86\%), pre-treatment neutrophil to lymphocyte ratio (> 3 in $55 \%, n=27$ ), sunitinib induced hypertension (45\%), and sunitinib dose reduction/treatment interruption (41\%). In clinical trial participants versus non-participants, RR was partial response/stable disease $80 \%(n=39)$ versus $74 \%(n=36)$, and progressive disease $20 \%(n=10)$ versus $26 \%(n=13)(p=0.63$; odds ratio, 1.2). The median PFS was 10 versus 11 months (hazard ratio [HR], 0.96; $p=0.84$ ), and the median OS 23 versus 24 months (HR, 0.97; $p=0.89$ ).

\section{Conclusion}

In mRCC patients treated with sunitinib, the outcome of clinical trial participants was similar to that of non-participants who received standard therapy. 


\section{Introduction}

Renal cell carcinoma (RCC) is the most common cancer of the kidney [1]. Approximately 20\%-30\% of patients are diagnosed with metastatic disease, and $70 \%-80 \%$ of patients are presented with localized or locally advanced disease at the time of diagnosis, which is potentially curable by radical surgical resection alone [2]. Among patients who undergo radical resection for localized disease, future metastatic disease develops in $20 \%-40 \%$ of the patient population [3].

An understanding of the pathogenesis of RCC at the molecular level, and randomized clinical trials, have established the standard role of the orally administered vascular endothelial growth factor receptor and platelet derived growth factor receptor inhibitor sunitinib for the treatment of advanced RCC [4].

Clinical trials are the standard way to evaluate the efficacy of new investigational therapies [5-7]. In various types of cancer (e.g., melanoma, ovarian cancer, lung cancer), data suggests that there exist a 'trial effect,' whereby clinical trials participants may have an improved outcome simply by participating in a clinical trial itself [5-10]. Positive effects of clinical trials participation may include improved routine care and treatment delivery, quality control, and close observation, leading to better patient compliance and clinician practice $[5,7,8,9,11]$

The effect of clinical trial participation on the outcome of treatment in metastatic RCC is poorly defined. This knowledge would be important, as treatment options and clinical trials with targeted therapies for patients with metastatic RCC have significantly expanded in recent years. Thus, we aimed to study the effect of clinical trial participation on the outcome of metastatic RCC patients treated with sunitinib.

\section{Materials and Methods}

\section{Study group}

We reviewed the records of patients (unselected cohort, international multicenter database) with evidence of metastatic RCC, who were treated with sunitinib between February 1, 2004, and December 31, 2013, in six centers across two different countries: the United States (Sidney Kimmel Comprehensive Cancer Center at Johns Hopkins, Baltimore, MD) and Israel (Institute of Oncology, Meir Medical Center, Kfar Saba; Department of Oncology, Asaf Harofe Medical Center, Zerifin; Department of Oncology, Rambam Medical Center, Haifa; Department of Oncology, Sheba Medical Center, Tel
Hashomer; Department of Oncology, Soroka University Medical Center, Beer-Sheva). Patients treated with sunitinib within clinical trials were identified and individually matched by clinicopathologic factors to patients treated with sunitinib as standard therapy out of a clinical trial. Patient data were retrospectively and personally collected by the investigator D.K. from electronic medical records and paper charts, including the following baseline clinical characteristics and known prognostic factors [12-19]: age, sex, pretreatment smoking status (active versus past/never), histology (clear cell versus non clear cell), past nephrectomy, the time interval from initial diagnosis to sunitinib treatment initiation, prior systemic therapies, number of metastases sites, presence of lung/liver/bone metastases, Eastern Cooperative Oncology Group (ECOG) performance status, hemoglobin level, corrected (for albumin) calcium level, pre-treatment neutrophil to lymphocyte ratio (NLR), sunitinib induced hypertension, and sunitinib dose reduction or treatment interruption. Data on the concomitant use of medications, including angiotensin system inhibitors (angiotensin converting enzyme inhibitors and angiotensin II receptor blockers) and bisphosphonates, was gathered from patients electronic medical records and paper charts, pharmacy records, and by contacting patients and other treating physicians as needed. The outcome data was last updated on December 31, 2013, including the objective response rate, progression free survival (PFS), and overall survival (OS). Patients who did not progress or die by December 31, 2013 were censored in PFS analysis or OS analysis, respectively.

\section{Sunitinib treatment}

All patients had objective disease progression on scans before starting sunitinib treatment. Sunitinib was administered orally, usually at a starting dose of $50 \mathrm{mg}$ once daily, in 6-week cycles consisting of 4 weeks of treatment followed by 2 weeks without treatment. On treatment dose reduction or treatment interruption were done for the management of adverse events, depending on their type and severity, in accordance to the standard guidelines. Treatment was continued until evidence of disease progression on scans, unacceptable adverse events, or death. Patient follow-up generally consisted of regular physical examinations and laboratory assessments (hematologic and serum chemical measurements), every 4-6 weeks, and imaging studies performed every 12-18 weeks.

\section{Treatment outcomes}

For the evaluation of response, the Response Evaluation Criteria in Solid Tumors (RECIST) ver. 1.1 was applied [19]. In patients with only bone metastases, only complete 
response, stable disease, or progressive disease were noted, not partial response [19]. The response was assessed by independent radiologists and treating physicians (while the patients were on treatment in each center, as part of standard patient follow-up). PFS was defined as the time from the initiation of sunitinib treatment until evidence of disease progression on scans or death of any cause. Overall survival was defined as the time from the initiation of sunitinib treatment to death of any cause.

\section{Statistical analysis}

Data was analyzed retrospectively. Patients in the groups of clinical trials participants and non-participants were individually matched by age, sex, RCC histology, prior nephrectomy, prior systemic therapy, sunitinib induced hypertension, sunitinib dose reduction/treatment interruption, the use of angiotensin system inhibitors, Heng risk, and pretreatment NLR.

Clinicopathologic characteristics and response rate were compared between clinical trials participants and nonparticipants, by chi-square test or Fisher's exact test for nominal data, and two-sample t test (or Mann-Whitney non-parametric test) for continuous measures. In all tests, a two tailed p-value of less than 0.05 was considered statistically significant. Cox proportional hazards regression model was used for comparison of PFS and OS between the two groups.

Furthermore, to determine if participation in a clinical trial is independently associated with treatment outcome, a univariate analysis (unadjusted) of association between each clinicopathologic factor and clinical outcome was performed for the entire patient cohort, using logistic regression for response rate and Cox regression model for survival outcomes (PFS and OS). Factors with significant association in the univariate analysis were included in multivariate Cox proportional hazards regression model to determine their independent effects.

PFS and OS times (probability and median) were estimated from Kaplan-Meier curve. Data were analyzed using SPSS ver. 21 (IBM Co., Armonk, NY).

\section{Regulatory considerations}

The research was carried out in accordance with the approval by the Institutional Review Board committee of our institutions.

\section{Results}

\section{Patient characteristics}

Forty-nine patients treated with sunitinib within clinical trials (NCT00083889, NCT00130897, NCT00444314) were identified and individually matched, via clinicopathologic factors, to 49 patients treated with sunitinib as the standard therapy out of a clinical trial. The distribution of clinicopathologic factors is shown in Table 1. Clinicopathologic factors used to individually match patients were age (median, 64 years), sex (male, 67\%), histology (clear cell, 86\%; $\mathrm{n}=42)$, past nephrectomy $(92 \%, \mathrm{n}=45)$, prior systemic therapy $(16 \%, \mathrm{n}=8$; including interferone $\mathrm{n}=5$ in clinical trials participants and $n=6$ in non-participants, or IL- $2 n=3$ in participants and $n=2$ in non-participants), sunitinib induced hypertension $(45 \%, \mathrm{n}=27)$, sunitinib dose reduction/treatment interruption $(41 \%, \mathrm{n}=20)$, use of angiotensin system inhibitors $(37 \%, \mathrm{n}=18)$, Heng risk stratification (favorable $25 \%, \mathrm{n}=12$; intermediate $59 \%, \mathrm{n}=29$; poor $16 \%, \mathrm{n}=8$ ), and pre-treatment NLR ( $\geq 3$ in 55\%, $\mathrm{n}=27$ ). The groups were balanced with regard to the presence of lung $(\mathrm{p}=0.62) /$ liver $(p=0.27) /$ bone $(p=0.67)$ metastases, number of metastatic sites $(\mathrm{p}=0.8)$, smoking status $(\mathrm{p}=0.97)$, presence of sarcomatoid component in tumor histology $(\mathrm{p}=0.84)$, and subsequent lines of therapy $(p=0.7$ and $p=0.85$ for subsequent second and third lines of therapy). In clinical trial participants, subsequent second line of therapy included sorafenib $(n=13)$, bevacizumab $(n=6)$, and everolimus $(n=5)$; moreover, subsequent third line of therapy included everolimus $(n=2)$, bevacizumab $(n=1)$, and sunitinib rechallenge $(n=1)$. In clinical trial non-participants, subsequent second line of therapy included temsirolimus $(n=7)$, everolimus $(n=5)$, sorafenib $(n=4)$, pazopanib $(n=3)$, and bevacizumab $(n=2)$; and subsequent third line of therapy included pazopanib $(n=2)$, and axitinib $(\mathrm{n}=1)$.

\section{Sunitinib treatment outcomes}

In clinical trial participants versus non-participants, objective response was partial response/ stable disease $80 \%(n=39)$ versus $74 \%(\mathrm{n}=36)$, and progressive disease at first imaging evaluation within the first 3 months $20 \%(n=10)$ versus $26 \%$ $(\mathrm{n}=13)$ ( $\mathrm{p}=0.63$; odds ratio, 1.2$)$ (Table 2$)$. The median PFS (Fig. 1) was 10 versus 11 months (hazard ratio [HR], 0.96; $\mathrm{p}=0.84$ ), and median OS (Fig. 2) 23 versus 24 months (HR, 0.97; $\mathrm{p}=0.89$ ) (Table 2). 
Table 1. Baseline patients characteristics stratified by status of clinical trial participation

\begin{tabular}{|c|c|c|c|}
\hline Characteristic & $\begin{array}{l}\text { Clinical trial participants } \\
\qquad(\mathrm{n}=49)\end{array}$ & $\begin{array}{c}\text { Matched clinical trial } \\
\text { non-participants }(n=49)\end{array}$ & p-value \\
\hline \multicolumn{4}{|l|}{ Age (yr) } \\
\hline Median (range) & $64(22-79)$ & $64(24-77)$ & 0.8 \\
\hline Mean \pm SD & $61 \pm 11$ & $62 \pm 10$ & \\
\hline \multicolumn{4}{|l|}{ Sex } \\
\hline Male & $33(67)$ & $33(67)$ & $>0.99$ \\
\hline Female & $16(33)$ & $16(33)$ & \\
\hline Tumor histology & & & $>0.99$ \\
\hline Clear cell histology & $42(86)$ & $42(86)$ & \\
\hline Non-clear cell & $7(14)$ & $7(14)$ & \\
\hline Presence of sarcomatoid component & $3(6)$ & $4(8)$ & 0.84 \\
\hline Past nephrectomy & $45(92)$ & $45(92)$ & $>0.99$ \\
\hline Prior systemic therapy & $8(16)$ & $8(16)$ & $>0.99$ \\
\hline Lung metastasis & $38(78)$ & $40(82)$ & 0.62 \\
\hline Liver metastasis & $12(24)$ & $17(35)$ & 0.27 \\
\hline Bone metastasis & $18(37)$ & $16(33)$ & 0.67 \\
\hline$\geq 2$ metastatic sites & $41(84)$ & $44(90)$ & 0.8 \\
\hline Sunitinib induced HTN & $27(45)$ & $27(45)$ & $>0.99$ \\
\hline Sunitinib dose reduction/treatment interruption & $20(41)$ & $20(41)$ & $>0.99$ \\
\hline Users of ASIs & $18(37)$ & $18(37)$ & $>0.99$ \\
\hline Users of bisphosphonates & $11(22)$ & $2(4)$ & 0.04 \\
\hline \multicolumn{4}{|l|}{ Heng risk stratification } \\
\hline Favorable & $12(25)$ & $12(25)$ & $>0.99$ \\
\hline Intermediate & $29(59)$ & $29(59)$ & \\
\hline Poor & $8(16)$ & $8(16)$ & \\
\hline Pre-treatment NLR $>3$ & $27(55)$ & $27(55)$ & $>0.99$ \\
\hline \multicolumn{4}{|l|}{ Smoking status } \\
\hline Never & $19(39)$ & $20(41)$ & 0.97 \\
\hline Past & $18(37)$ & $18(37)$ & \\
\hline Active & $12(24)$ & $11(22)$ & \\
\hline \multicolumn{4}{|l|}{ Subsequent therapy lines } \\
\hline Second line & $24(49)$ & $21(43)$ & 0.7 \\
\hline Third line & $4(8)$ & $3(6)$ & 0.85 \\
\hline
\end{tabular}

Values are presented as number (\%) unless otherwise indicated. SD, standard deviation; HTN, hypertension; ASIs, angiotensin system inhibitors; NLR, neutrophil to lymphocyte ratio.

\section{Univariate analysis (entire patient cohort, $n=98$ ) of factors associated with PFS and OS}

Smoking status (HR, 3.35; $\mathrm{p}<0.001$ for active vs. never / past smokers), Heng risk (HR, 1.23 and 2.5; $\mathrm{p}=0.41$ and $\mathrm{p}=0.005$, for favorable and intermediate versus poor risk, respectively), use of angiotensin system inhibitors (HR, 0.6 for yes vs. no; $\mathrm{p}=0.024)$, past nephrectomy (HR, 0.41 for yes vs. no; $\mathrm{p}=0.014)$, sunitinib induced hypertension ( $\mathrm{HR}, 0.57$ for yes vs no; $\mathrm{p}=0.009)$, non-clear cell histology (HR, 2.8 vs. clear cell histology; $p=0.001)$, and pre-sunitinib treatment NLR $>3$ (HR, 2.5 for $>3$ vs. $\leq 3 ; \mathrm{p}<0.001)$ were individually associated with PFS. The status of clinical trial participation (yes vs. no) was not associated with PFS (HR, 1.1; $\mathrm{p}=0.7$ ).

Smoking status (HR, 3; $\mathrm{p}<0.001$ for active vs. never/past smokers), Heng risk (HR, 1.38 and 2.13; $\mathrm{p}=0.23$ and $\mathrm{p}=0.032$, for favorable and intermediate versus poor risk, respectively), past nephrectomy (HR, 0.4 for yes vs. no; $\mathrm{p}=0.012$ ), non-clear cell histology (HR, 2 vs. clear cell histology; $p=0.023$ ), sunitinib induced hypertension (HR, 0.59 for yes vs. no; $\mathrm{p}=0.019$ ), and pre-sunitinib treatment NLR $>3$ (HR, 1.8 for $>3$ vs. $\leq 3 ; p=0.009$ ) were individually associated with OS. Status of clinical trial participation (yes vs. no) was not associated with PFS (HR, 1.15; $\mathrm{p}=0.48)$. 
Table 2. Sunitinib treatment outcome stratified by status of clinical trial participation

\begin{tabular}{|c|c|c|c|c|}
\hline Treatment outcome & $\begin{array}{l}\text { Clinical trial participants } \\
\qquad(\mathrm{n}=49)\end{array}$ & $\begin{array}{l}\text { Matched clinical trial } \\
\text { non-participants }(n=49)\end{array}$ & p-value & HR $(95 \% \mathrm{CI})$ \\
\hline Response rate & & & & Odds ratio, 1.2 \\
\hline Partial response & $26(53)$ & $20(41)$ & 0.63 & \\
\hline Stable disease & $13(27)$ & $16(33)$ & & \\
\hline $\begin{array}{l}\text { Disease progression within } 12 \text { weeks of } \\
\text { the start of sunitinib }\end{array}$ & $10(20)$ & $13(26)$ & & \\
\hline Median PFS (mo) & 10 & 11 & 0.84 & $0.96(0.68-1.19)$ \\
\hline Median OS (mo) & 23 & 24 & 0.89 & $0.97(0.72-1.13)$ \\
\hline
\end{tabular}

Values are presented as number $(\%)$ unless otherwise indicated. HR, hazard ratio; CI, confidence interval; PFS, progression free survival; OS, overall survival.

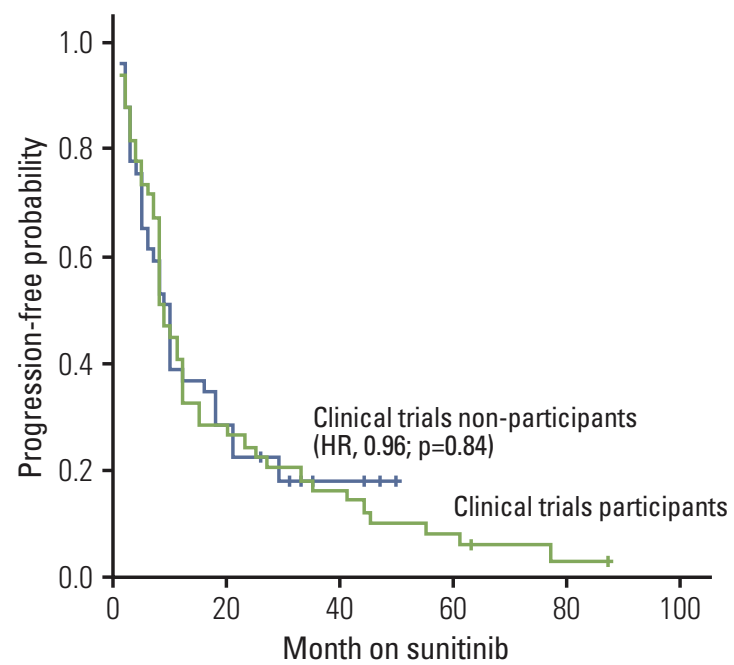

Fig. 1. Kaplan-Meier estimates of progression-free survival stratified by status of clinical trials participation. HR, hazard ratio.

\section{Multivariate analysis (entire patient cohort, $n=98$ ) of factors associated with PFS and OS}

Factors independently associated with PFS were smoking status (HR, 2.18; $\mathrm{p}=0.009$, for active vs. never / past smokers), non-clear cell histology (HR, 3 vs. clear cell histology; $\mathrm{p}=0.008$ ), use of angiotensin system inhibitors (HR, 0.56 for yes vs. no; $\mathrm{p}=0.03)$, and pre-treatment NLR $>3(\mathrm{HR}, 1.95$ for $>3$ vs. $\leq 3 ; \mathrm{p}=0.015)$. Factors that were independently associated with OS were smoking status (HR, 2.9; $\mathrm{p}=0.001$, for active vs never/ past smokers), non-clear cell histology (HR, 2.1 vs. clear cell histology; $\mathrm{p}=0.032)$, and pre-treatment NLR $>3$ (HR, 1.4 for $>3$ vs. $\leq 3 ; \mathrm{p}=0.02)$.

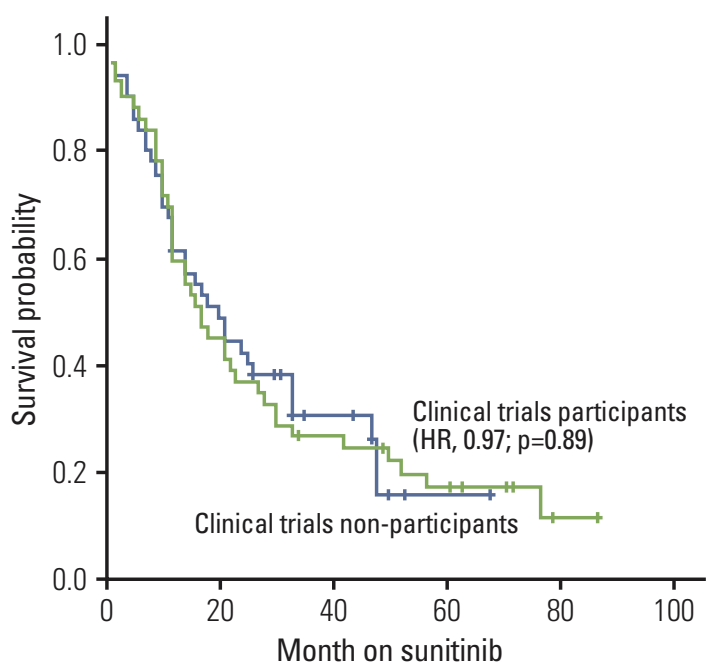

Fig. 2. Kaplan-Meier estimates of overall survival stratified by status of clinical trials participation. HR, hazard ratio.

\section{Discussion}

In the present study, we did not find a 'clinical trial effect' in patients with metastatic RCC treated with sunitinib. The outcome of clinical trial participants was similar to that of matched patients treated out of a trial, in terms of objective response, PFS, and OS. Furthermore, in a univariate-multivariate analysis of the entire patient cohort (trial participants plus non-participants), the status of clinical trial participation was not associated with treatment outcome.

A positive effect of clinical trial participation on treatment outcome, may be explained by multiple factors, including a 
protocol effect (the way therapy is given), care effect (incidental aspects of care), Hawthorne effect (changes in health care practitioners and patients behavior due to the knowledge that they are under monitoring), placebo effect, treatment (per clinical trial) in high-volume centers (per clinical trial) versus non-academic institutions (i.e., participation in clinical trials may represent a quality characteristic of medical centers. Thus, healthcare systems that are active in clinical trials may deliver better outcomes for patients than healthcare systems that are inactive in trials) $[9-11,20]$. Another potential association between clinical trial participation and outcome may also arise if clinicians who tend to recruit to trials also tend to be more competent clinicians [8].

However, some studies found only a weak evidence to suggest that clinical trials participation leads to a positive effect on the outcome of participants [8,9,20-22].

Furthermore, other studies found a trial effect only when the investigational treatment arm of a clinical trial was more efficacious than the standard treatment arm [20]. Moreover, improvement in outcomes of patients participating in clinical trials, as reported by some studies, might be related to bias from methodological difficulties as data collection, i.e., retrospective analysis, patient selection (e.g., exclusion of patients with coexisting medical conditions), a better followup of patients treated per clinical trials than out of trials, and failure to publish studies reporting negative trial effects $[9,20]$.

Various reasons might be put forward to explain the present study findings, of similar outcomes between clinical trial participants and non-participants. First, all non-participants were treated in academic medical centers. It is possible that institutional standardization of care (e.g., regular follow-up by a dedicated nurse who specialized in the management of sunitinib toxicity), may provide the same benefit as treatment, thus eliminating the potential for the participation effects [20]. Second, our patients were treated with an oral targeted agent. In most studies reporting clinical trial effect, patients were treated with intravenous chemotherapy and radiation therapy. To the best of our knowledge, the effect of clinical trial participation on the outcome of modern targeted therapies is poorly defined. Finally, some data suggests that when stratified by tumor type, the beneficial effect of trial enrollment may be seen only in selected types, such as breast, lung and colon cancer, and may not be seen in other types, such as melanoma, pancreatic and hepatobiliary cancers [23].

Our study has some limitations. First, this is a multicenter retrospective study that represents an unselected heterogeneous cohort of patients that were treated with sunitinib, including all histologic variants of RCC, and patients who were treatment naïve and those with a history of prior therapy. Moreover, our data do not exclude the possibility of a trial effect at other institutions. Nonetheless, the outcome of the present study patient population (i.e., median PFS of 10-11 months, and median OS of 23-24 months) is similar to the previously published data on patients with metastatic RCC that are treated with sunitinib [19]. Second, we are unable to exclude the possibility that unequal distribution of unidentified clinicopathologic parameters in our patient cohort may have biased the observed results. Third, the total number of 98 patients is relatively small. Thus, our study might have been underpowered to show a difference in the outcome. Other clinicopathologic factors, including status of clinical trial participation, that were not found to be significantly associated with disease progression in the present study might have been important in a larger patient cohort.

Fourth, whether our findings are specific to sunitinib or generalizable to other tyrosine kinase inhibitors is not known.

Despite these limitations, our clinical observation on the status of clinical trial participation does not impact the outcome of sunitinib treatment in metastatic RCC an may contribute to treatment decisions, patient selection, and clinical trials design. Given the above evidence, discussion between clinicians and mRCC patients about participation in a clinical trial should focus on the improvement of future therapy, and not the direct benefit of research participation. Physicians should not imply a survival benefit when counseling metastatic RCC patients about entering clinical trials.

Further prospective studies may be warranted, to test and confirm our hypothesis generating observation in larger patient cohorts, to define the association between clinical trial participation and outcome in different subgroups of patients (e.g., according to risk by prognostic models, clear cell versus non-clear cell histology, and first line versus advanced line treatment).

\section{Conclusion}

We investigated the effect of clinical trial participation on outcome of patients with mRCC who were treated with sunitinib. After accounting for other clinicopathologic factors, we were unable to show that participation in a clinical trial may in itself provide an outcome advantage to these patients.

The present study may improve confidence that the trial treatment effects will translate to the real-world setting. Discussion between clinicians and $\mathrm{mRCC}$ patients regarding participation in a clinical trial should focus on the improvement of future therapy rather than the direct outcome of research participation. 


\section{Conflicts of Interest}

Conflict of interest relevant to this article was not reported.

\section{References}

1. Li L, Kaelin WG Jr. New insights into the biology of renal cell carcinoma. Hematol Oncol Clin North Am. 2011;25:667-86.

2. Massari F, Bria E, Maines F, Milella M, Giannarelli D, Cognetti F, et al. Adjuvant treatment for resected renal cell carcinoma: are all strategies equally negative? Potential implications for trial design with targeted agents. Clin Genitourin Cancer. 2013;11:471-6.

3. Herrmann E, Weishaupt C, Poppelmann B, Hillgruber C, Puhse G, Krabbe LM, et al. New tools for assessing the individual risk of metastasis in renal cell carcinoma. Clin Exp Metastasis. 2013;30:215-24.

4. Motzer RJ, Hutson TE, Tomczak P, Michaelson MD, Bukowski $\mathrm{RM}$, Rixe $\mathrm{O}$, et al. Sunitinib versus interferon alfa in metastatic renal-cell carcinoma. N Engl J Med. 2007;356:115-24.

5. Tanai C, Nokihara H, Yamamoto S, Kunitoh H, Yamamoto N, Sekine I, et al. Characteristics and outcomes of patients with advanced non-small-cell lung cancer who declined to participate in randomised clinical chemotherapy trials. Br J Cancer. 2009;100:1037-42.

6. Field KM, Drummond KJ, Yilmaz M, Tacey M, Compston D, Gibbs $\mathrm{P}$, et al. Clinical trial participation and outcome for patients with glioblastoma: multivariate analysis from a comprehensive dataset. J Clin Neurosci. 2013;20:783-9.

7. Braunholtz DA, Edwards SJ, Lilford RJ. Are randomized clinical trials good for us (in the short term)? Evidence for a "trial effect". J Clin Epidemiol. 2001;54:217-24.

8. Goyal J, Nuhn P, Huang P, Tyagi P, Oh D, Carducci MA, et al. The effect of clinical trial participation versus non-participation on overall survival in men receiving first-line docetaxelcontaining chemotherapy for metastatic castration-resistant prostate cancer. BJU Int. 2012;110(11 Pt B):E575-82.

9. Peppercorn JM, Weeks JC, Cook EF, Joffe S. Comparison of outcomes in cancer patients treated within and outside clinical trials: conceptual framework and structured review. Lancet. 2004;363:263-70.

10. Rajappa S, Gundeti S, Uppalapati S, Jiwatani S, Abhyankar A, $\mathrm{Pal} \mathrm{C}$, et al. Is there a positive effect of participation on a clinical trial for patients with advanced non-small cell lung cancer? Indian J Cancer. 2008;45:158-63.

11. Rochon J, du Bois A. Clinical research in epithelial ovarian cancer and patients' outcome. Ann Oncol. 2011;22 Suppl 7:vii16-9.

12. Patil S, Figlin RA, Hutson TE, Michaelson MD, Negrier S, Kim ST, et al. Prognostic factors for progression-free and overall survival with sunitinib targeted therapy and with cytokine as first-line therapy in patients with metastatic renal cell carcinoma. Ann Oncol. 2011;22:295-300.

13. Motzer RJ, Bukowski RM, Figlin RA, Hutson TE, Michaelson
MD, Kim ST, et al. Prognostic nomogram for sunitinib in patients with metastatic renal cell carcinoma. Cancer. 2008;113: $1552-8$.

14. Choueiri TK, Plantade A, Elson P, Negrier S, Ravaud A, Oudard S, et al. Efficacy of sunitinib and sorafenib in metastatic papillary and chromophobe renal cell carcinoma. J Clin Oncol. 2008;26:127-31.

15. Rini BI, Cohen DP, Lu DR, Chen I, Hariharan S, Gore ME, et al. Hypertension as a biomarker of efficacy in patients with metastatic renal cell carcinoma treated with sunitinib. J Natl Cancer Inst. 2011;103:763-73.

16. Heng DY, Xie W, Regan MM, Warren MA, Golshayan AR, Sahi C, et al. Prognostic factors for overall survival in patients with metastatic renal cell carcinoma treated with vascular endothelial growth factor-targeted agents: results from a large, multicenter study. J Clin Oncol. 2009;27:5794-9.

17. Keizman D, Huang P, Eisenberger MA, Pili R, Kim JJ, Antonarakis ES, et al. Angiotensin system inhibitors and outcome of sunitinib treatment in patients with metastatic renal cell carcinoma: a retrospective examination. Eur J Cancer. 2011;47:1955-61.

18. Keizman D, Ish-Shalom M, Huang P, Eisenberger MA, Pili R, Hammers $\mathrm{H}$, et al. The association of pre-treatment neutrophil to lymphocyte ratio with response rate, progression free survival and overall survival of patients treated with sunitinib for metastatic renal cell carcinoma. Eur J Cancer. 2012;48:202-8.

19. Keizman D, Gottfried M, Ish-Shalom M, Maimon N, Peer A, Neumann A, et al. Active smoking may negatively affect response rate, progression-free survival, and overall survival of patients with metastatic renal cell carcinoma treated with sunitinib. Oncologist. 2014;19:51-60.

20. Koschmann C, Thomson B, Hawkins DS. No evidence of a trial effect in newly diagnosed pediatric acute lymphoblastic leukemia. Arch Pediatr Adolesc Med. 2010;164:214-7.

21. Abu-Hejleh T, Chrischilles EA, Halfdanarson TR, Simon C, Pendergast JF, Jiang D, et al. The effect of receiving treatment within a clinical trial setting on survival and quality of care perception in advanced stage non-small cell lung cancer. Am J Clin Oncol. 2014 Mar 13 [Epub]. http://dx.doi.org/10.1097/ COC.0000000000000029.

22. Unger JM, Barlow WE, Martin DP, Ramsey SD, Leblanc M, Etzioni R, et al. Comparison of survival outcomes among cancer patients treated in and out of clinical trials. J Natl Cancer Inst. 2014;106:dju002.

23. Chow CJ, Habermann EB, Abraham A, Zhu Y, Vickers SM, Rothenberger DA, et al. Does enrollment in cancer trials improve survival? J Am Coll Surg. 2013;216:774-80. 